Recebido em 03/2020. Aceito para publicação em 08/2020.

\title{
AVALIAÇÃO CLÍNICA E MORFOLÓGICA DO TENDÃO DO CALCÂNEO: ESTUDO ULTRASSONOGRÁFICO DE SUJEITOS PATOLÓGICOS E SADIOS
}

\section{CLINICAL AND MORPHOLOGICAL EVALUATION OF THE CALCANEUS TENDON: ULTRASONOGRAPHY STUDY OF HEALTHY AND PATHOLOGICAL SUBJECTS}

\author{
Ana Paula Pinto 1 \\ Mário Oliveira Lima ${ }^{2}$ \\ Patrícia Sardinha Leonardo ${ }^{3}$ \\ Douglas Peretta Barbaroto 4 \\ Natália Galvão Rocha Moreira ${ }^{5}$ \\ Ary Teixeira Breguez de Oliveira ${ }^{6}$ \\ Fernanda Púpio Silva Lima ${ }^{7}$ \\ Jon Joensen ${ }^{8}$ \\ Jan Magnus Bjordal ${ }^{9}$
}

Rodrigo Alvaro Brandão Lopes-Martins ${ }^{10}$

Resumo: O tendão do calcâneo (TC) é o mais forte tendão do corpo humano, com grande capacidade de suportar carga. O TC é vulnerável a lesões por esforço repetitivo por receber muita carga, responsável por $18 \%$ de todas as lesões no esporte. O ultrassom (US) diagnóstico é uma técnica barata, dinâmica e rápida para avaliação de tecidos tendíneos, que pode ser associada a testes clínicos para diagnóstico de tendinopatias. Considerando que a simetria do organismo está relacionada com bom estado geral de saúde, objetivou-se neste trabalho avaliar a morfologia e aspectos clínicos do TC de indivíduos com e sem tendinopatia do TC. Participaram do estudo 28 indivíduos: 15 no grupo controle (GC) e 13 no grupo tendinopatia (GT). Os participantes passaram por avaliações específicas do TC: Exame de US, testes à palpação e clínicos. Os sujeitos do GC apresentam média de 0,407cm e 0,389cm da espessura do TC (TC direito e TC esquerdo), e não foram demonstradas anormalidades que indicassem inflamação nas imagens de US e nos testes clínicos. Nos indivíduos do GT, os valores da espessura do TC foram maiores, atingindo até $0,563 \mathrm{~cm}$, além de apresentarem alterações nas imagens de US para inflamação. A diferença da espessura do TC entre membros dos indivíduos do GC foi de $10 \%$, enquanto no GT foi de $22 \%$. Por meio dos resultados, sugere-se que diferenças da espessura do TC superiores a $20 \%$ indiquem a presença de tendinopatia, comprovadas pelos achados nas imagens de ultrassom e pelos resultados positivos dos testes à palpação e clínicos.

\footnotetext{
${ }^{1}$ Laboratório de Engenharia de Reabilitação Sensório Motora, Universidade do Vale do Paraíba - Univap, Brasil. E-mail: apaula@outlook.com.br.

2 Laboratório de Engenharia de Reabilitação Sensório Motora, Universidade do Vale do Paraíba - Univap, Brasil. E-mail: mol@univap.br.

${ }^{3}$ Laboratório de Biofotônica e Terapêutica Experimental, Universidade do Vale do Paraíba - Univap, Brasil. Email: pssardinha@yahoo.com.br.

${ }^{4}$ Laboratório de Biofotônica e Terapêutica Experimental, Universidade do Vale do Paraíba - Univap, Brasil. Email: barbaroto@hotmail.com.

${ }^{5}$ Laboratório de Biofotônica e Terapêutica Experimental, Universidade do Vale do Paraíba - Univap, Brasil. Email: ftnatalia.galvao@gmail.com.

${ }^{6}$ Laboratório de Biofotônica e Terapêutica Experimental, Universidade do Vale do Paraíba - Univap, Brasil. Email: ary14teixeira@gmail.com.

${ }^{7}$ Laboratório de Engenharia de Reabilitação Sensório Motora, Universidade do Vale do Paraíba - Univap, Brasil. E-mail: fpupio@univap.br.

8 Department of Physiotherapy, University of Bergen, Noruega. E-mail: Jon.Joensen@uib.no.

9 Department of Physiotherapy, University of Bergen, Noruega. E-mail: Jan.Bjordal@uib.no.

10 Post-graduation Program in Human Movement and Rehabilitation, Centro Universitário UniEvangélica, Brasil. E-mail: ralopesmartins@gmail.com.
} 
Palavras-chave: Ultrassom de imagem; tendão de Aquiles; tendão do calcâneo; tendinopatia; tendinite.

Abstract: Calcaneal tendon (CT) is the strongest tendon in the human body, with a great capacity to support load. CT is vulnerable to repetitive strain injuries since it receives too much load, responsible for $18 \%$ of all injuries in sports. Diagnostic ultrasound (US) is a cheap, dynamic and fast technique for assessing tendon tissues, which can be associated with clinical tests for the diagnosis of tendinopathies. Considering that the symmetry of the organism is related to good general health, the aim of this study was to evaluate the morphology and clinical aspects of CT in individuals with and without CT tendinopathy. 28 individuals participated in the study: 15 in the control group (CG) and 13 in the tendinopathy group (TG). The participants underwent specific CT assessments: US examination, palpation and clinical tests. The subjects of the CG had an average of $0.407 \mathrm{~cm}$ and $0.389 \mathrm{~cm}$ for CT thickness (right CT and left CT), and no abnormalities were shown that would indicate inflammation in the US images and in clinical tests. In the TG individuals, the CT thickness values were higher, reaching up to $0.563 \mathrm{~cm}$, in addition to showing changes in the US images for inflammation. The difference in CT thickness among members of the individuals in CG was $10 \%$, while in the GT it was 22\%. By the results, it is suggested that differences in CT thickness greater than $20 \%$ indicate the presence of tendinopathy, confirmed by the findings in the ultrasound images and by the positive results of the palpation and clinical tests.

Keywords: Ultrasound imaging; Achilles tendon; Calcaneus tendon; tendinopathy; tendonitis.

\section{INTRODUÇÂO}

As doenças musculoesqueléticas correspondem a um grupo amplo de alterações relacionadas a inflamação e degeneração de músculos, tendões, ligamentos, articulações e cartilagens, as quais podem resultar em sintomas álgicos e levar a limitação funcional. Estas fazem parte das doenças crônicas não transmissíveis e que acarretam grandes impactos socioeconômicos (SOUZA; SANTANA, 2011).

A tendinopatia do calcâneo ou de Aquiles é um distúrbio entre as doenças musculoesqueléticas que afeta o membro inferior. Em atletas, é responsável por 18\% de todas as lesões no esporte, com incidência cumulativa ao longo da vida de $24 \%$. Apesar de ser afetado em menor escala, indivíduos não-atléticos também são acometidos. A tendinopatia do calcâneo é a sexta razão mais comum para consultas não traumáticas de pé e tornozelo (MENZ et al. 2010; MUNTEANU, 2015).

O tendão do calcâneo (TC) é o mais largo e forte tendão do corpo humano, com a maior capacidade de suportar carga (O'BRIEN, 2005; KRAEUTLER; PURCELL; HUNT, 2017) e é biologicamente importante para o movimento mais funcional e rápido, sendo responsável pelos estágios finais do passo na marcha (RAMACHANDRAM, 2007). A força gerada no TC durante o exercício pode atingir 12 vezes o peso corporal, tornando o TC vulnerável a lesões por esforço repetitivo (BENJAMIN et al., 2006).

A Tendinopatia é uma síndrome clínica relacionada ao tendão e tecidos ao redor, podendo ser caracterizada por inflamação, deformação, microrrupturas ou rupturas. Inclui os distúrbios tendinose e tendinite, que resultam muitas vezes em dor e sensibilidade, bem como uma perda de força biomecânica e amplitude de movimento nas estruturas anatômicas envolvidas (MAFFULLI; SHARMA; LUSCOMBE, 2004; RIO et al., 2014). 
O sintoma mais comum é a dor. Edema, sensibilidade no local, crepitação também são comuns nos indivíduos com a patologia. Outras queixas, além da dor, estão relacionadas com o comprometimento da funcionalidade, como o a diminuição do desempenho nos esportes e nas atividades de vida diária, diminuição da amplitude de movimento e fraqueza muscular (MAFFULLI; SHARMA; LUSCOMBE, 2004; RYAN et al., 2009).

A etiologia da tendinopatia é considerada multifatorial, com fatores intrínsecos e extrínsecos envolvidos (MAFFULLI; SHARMA; LUSCOMBE, 2004). A carga excessiva nos tendões por tempo prolongado (overuse) é a causa mais comum de lesão do TC (COOK; KHAN; PURDAM, 2002; RIO et al., 2014). É comum que indivíduos sedentários e até mesmo atletas, não realizem a prevenção, por isso o diagnóstico precoce e o tratamento eficaz da tendinopatia do TC são essenciais para evitar um pior prognóstico, como a ruptura do tendão.

O diagnóstico da tendinopatia do TC é baseado principalmente na história, no exame clínico detalhado, e em exame de imagem. Atualmente, os exames de imagem mais utilizados para diagnóstico são o ultrassom e a ressonância magnética (JÓZSA; KANNUS, 1997; MARTIN et al., 2018; CHINESE SOCIETY OF SPORTS MEDICINE, 2019).

A ultrassonografia é uma técnica de imagem rápida, de baixo custo, que não emite radiação e não invasiva. O alto contraste acústico dos tendões com os tecidos ao redor, permite a avaliação ultrassonográfica. Esta técnica permite que os exames sejam mais dinâmicos e com feedback em tempo real. É possível realizar a análise anatômica, morfológica e alguns aparelhos com o modo Doppler, estudar a dinâmica circulatória (KHARATE; CHANCE-LARSEN, 2012; CHINESE SOCIETY OF SPORTS MEDICINE, 2019).

A simetria do organismo está relacionada com um bom estado geral de saúde. Do contrário, a assimetria de algumas estruturas do corpo humano pode ser refletida negativamente no desenvolvimento, como em algumas doenças genéticas cromossômicas, ou ser um marcador de uma possível condição patológica (PAWLOWSKI et al., 2018). Por isso, o objetivo deste trabalho foi avaliar e comparar a morfologia e aspectos clínicos do TC de indivíduos com e sem tendinopatia do calcâneo para traçar um perfil de normalidade e patológico de indivíduos sem e com tendinopatia do calcâneo.

\section{MATERIAL E MÉTODOS}

\subsection{Desenho Experimental e Amostra}

Estudo analítico, transversal e de cunho quali/quantitativo. Participaram voluntários com e sem tendinopatia do tendão calcâneo. 


\subsection{Critérios de Inclusão e Exclusão}

Para o grupo tendinopatia, os critérios de inclusão foram: Tendinopatia do TC unilateral ou bilateral com duração mínima de três meses, ambos os gêneros, idade entre 18-80 anos, sujeitos ativos ou sedentários e aparência de ultrassonografia patológica do TC, com tendão espessado. Quanto aos critérios de exclusão, foram: cirurgia anterior no TC, injeção de cortisona nos últimos 6 meses, doença inflamatória sistêmica, sutura / cirurgia prévia do TC, gravidez ou hipercolesterolemia familiar (pode provocar o xantoma do TC).

Para o grupo controle, os critérios de inclusão adotados foram: TC saudável, ambos os gêneros, idade entre 18-80 anos e sujeitos ativos ou sedentários. E os critérios de exclusão envolveram: doenças ou injúrias musculoesqueléticas em membros inferiores.

Todos os participantes foram instruídos a evitar o uso de medicamentos antiinflamatórios não esteróides (AINEs) uma semana antes da avaliação.

\subsection{Local de Estudo}

Este estudo foi realizado junto ao Laboratório de Engenharia de Reabilitação Sensório Motora pertencente ao Instituto de Pesquisa e Desenvolvimento (IP\&D) da Universidade do Vale do Paraíba (UNIVAP) e ao Centro de Práticas Supervisionadas pertencente à Faculdade de Ciências da Saúde (FCS) da UNIVAP.

\subsection{Aspectos Éticos do Estudo}

O presente projeto de pesquisa foi submetido à análise do Comitê de Ética e Pesquisa (CEP) através da Plataforma Brasil e foi aprovado sob o protocolo CAAE: 94820218.2.0000.5503.

Este estudo foi conduzido de acordo com a resolução no 466/2012 do Conselho Nacional de Saúde (CNS). Todos os voluntários foram previamente esclarecidos e orientados sobre os procedimentos. Após aceitação plena, todos os participantes assinaram o Termo de Consentimento Livre e Esclarecido.

\subsection{Riscos e Benefícios}

Os riscos desta pesquisa, ainda que classificados como baixos, incluem constrangimento, dor durante o teste e queda durante a locomoção do participante pelo local de pesquisa. No entanto, para minimizar a ocorrência dos riscos, os pesquisadores envolvidos na pesquisa foram profissionais responsáveis, orientados a atuar com ética, respeito e compromisso com os voluntários. Para reduzir o risco de queda, o participante sempre foi acompanhado por dois pesquisadores para dar suporte. E quanto relatado dor, os testes foram realizados com cautela e no limite de cada voluntário.

E como benefício, os voluntários tiveram a possibilidade de obter melhor conhecimento sobre a condição atual do TC e contribuição para o meio científico acerca 
de um novo recurso para identificação de patologias inflamatórias.

\subsection{Grupos do Estudo}

O presente estudo teve em sua composição o total de 28 participantes, divididos em dois grupos:

Grupo Tendinopatia (GT): $n=13$. Esses indivíduos eram portadores de tendinopatia do TC bilateral $(n=7)$ ou unilateral $(n=6)$.

Grupo Controle (GC): n=15. A tendinopatia do TC ou outras injúrias do sistema musculoesquelético em membros inferiores eram ausentes nos voluntários deste grupo.

\subsection{Coleta de Dados}

A bateria de avaliações para cada voluntário ocorreu em um dia, em que eles passaram pela anamnese, avaliação do exame de ultrassonografia e por testes físicos, respectivamente nesta ordem, para que um teste ou exame não interferisse no outro. $A$ seguir, serão comentadas com mais detalhes as avaliações realizadas.

\subsubsection{Anamnese e Ultrassom de Imagem}

Durante o período de acomodação do participante no ambiente da avaliação, foi realizada a anamnese a respeito de informações pessoais, perguntas sobre os hábitos de vida e histórico (ou não) sobre a tendinopatia do TC. Na coleta das imagens de ultrassom, foi utilizada uma sala com a luminosidade reduzida, para facilitar a visualização durante a captação das imagens.

Para a avaliação estrutural da disposição das fibras, limites do tendão, presença ou ausência de edema, calcificação e irrigação intratendínea, utilizou-se o aparelho de ultrassom portátil (Mindray M6®), com tela de 19", que foi operado nos Modos B, Color e Doppler com imagens de alta definição, resolução CrossXBeam e imagens harmônicas codificadas. Foi utilizado um transdutor/probe linear (L14-6NS) com ajuste para a frequência $12 \mathrm{MHz}$ e gel condutor a base de água com meio de contato e assim visualizar as estruturas com maior clareza. O aparelho possui uma função que possibilita a medição precisa e escalonada em centímetros.

A posição de coleta foi idêntica à avaliação da termografia, com o voluntário deitado em decúbito ventral, com os pés levemente para fora da maca e com os membros inferiores relaxados (tornozelo em posição neutra).

Primeiramente, foi realizado o cadastro do participante no sistema do aparelho e depois, foram coletadas imagens de ambos os membros, no corte longitudinal e transversal. Padronizou-se a superfície do osso calcâneo como ponto de referência, em que deveria estar presente no lado esquerdo da tela. A marcação de referência na probe 
(figura 1) seguia o padrão de ser direcionada ao osso calcâneo e virada para cima para obter a imagem no corte longitudinal (figura 2A) e as imagens no corte transversal, o ponto de referência era voltado para a parte medial do voluntário (figura $2 \mathrm{~B}$ ). As imagens foram nomeadas de acordo com o lado do membro e realizadas medidas do comprimento e espessura, função presente no próprio aparelho (HASLERUD et al., 2017).

Figura 1 - Ponto de referência da probe para orientação.

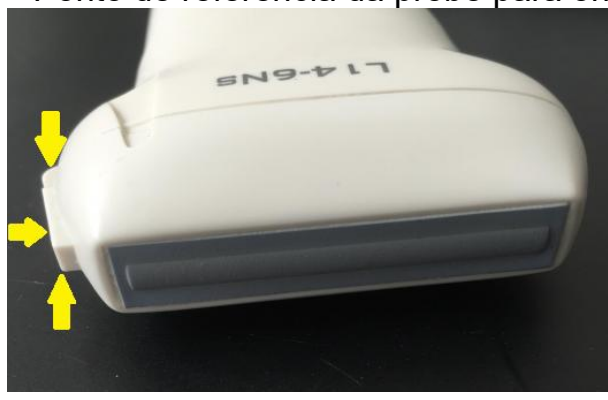

Fonte: Os autores.

Figura 2 - Posição da probe para coleta da imagem.

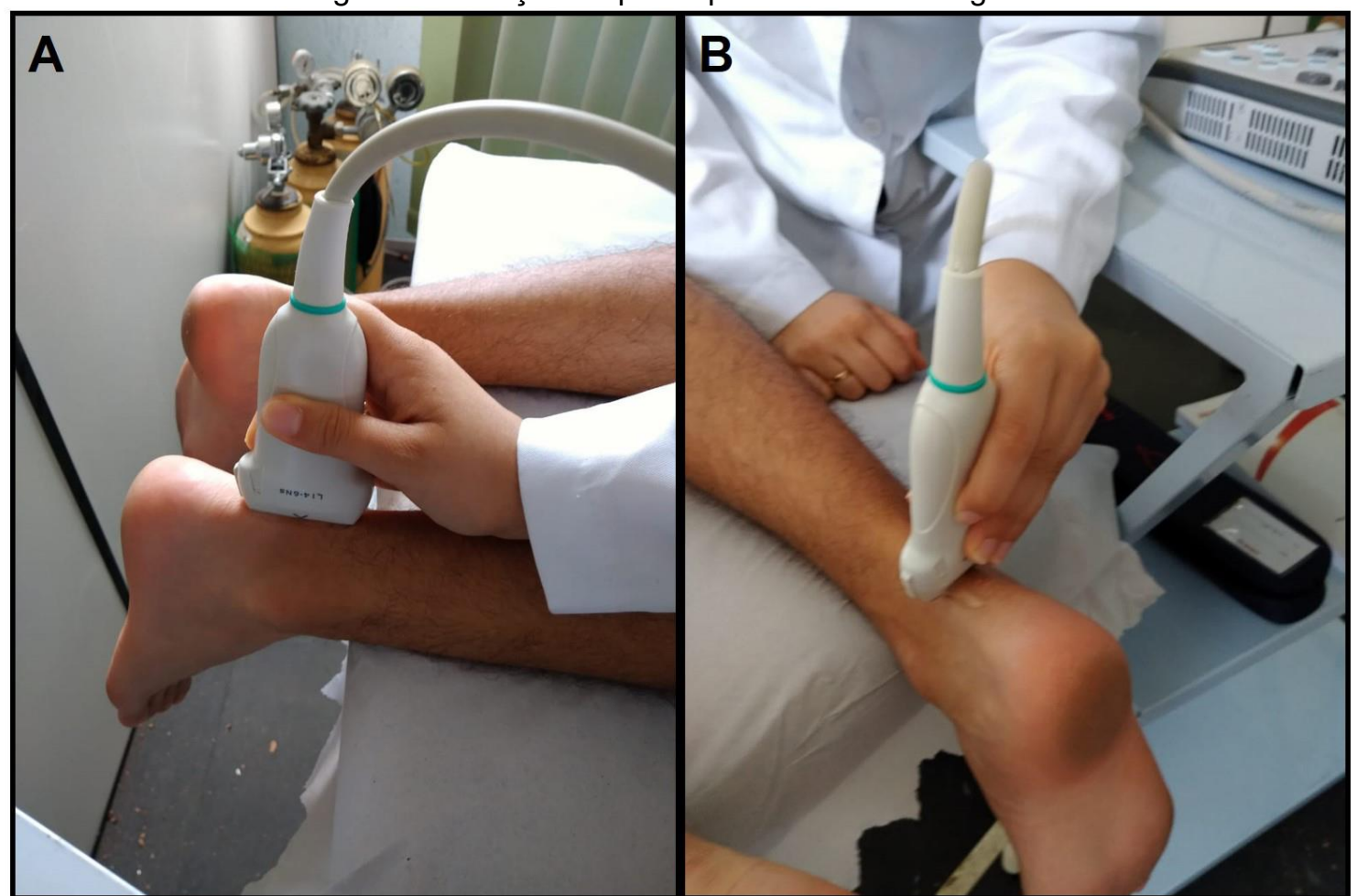

Legenda: A= Eixo longitudinal (ponto de referência voltado para o osso calcâneo); B= Eixo transversal (ponto de referência sentido linha mediana do participante).

Fonte: Os autores.

Considerou-se para a estatística a medida da espessura no corte longitudinal, ausência ou presença de edema em ambos os cortes, ausência ou presença de irrigação intratendínea no corte transversal com modo PW ativado (Doppler), e levou-se em consideração para análise de outros achados ultrassonográficos (irregularidade das fibras ou borda do tendão e calcificação) o corte transversal e longitudinal. As imagens 
foram salvas em formato JPEG, transferidas para um computador através de um pendrive USB, as quais foram salvas em um computador e analisadas com cautela.

\subsubsection{Crepitação, Espessura e Ternura (sensibilidade) à Palpação}

A degeneração das fibras do tecido conjuntivo, devido ao processo inflamatório, causa hiperemia, espessamento na área com a infiltração de células inflamatórias e o exsudato fibrinoso envolvendo o tendão e causa crepitação (estalido). Na inflamação crônica, as fibras lesionadas podem calcificar devido a constante tentativa de cicatrização. Com os depósitos de cálcio nas lesões há perda do tecido conjuntivo e da elasticidade e causar dor e sensibilidade (ternura) à palpação (KHAN et al., 1999; VIEIRA et al., 2010; MASCl et al., 2016).

$\mathrm{Na}$ avaliação, realizamos a mensuração dos sinais de espessamento, crepitação e maciez por meio da palpação do tendão com o voluntário deitado em decúbito ventral, com os joelhos estendidos e os tornozelos nas posições neutra para os movimentos de flexão plantar e dorsiflexão.

O resultado era considerado positivo para crepitação se apresentasse o estalido durante o movimento; positivo para espessamento se observasse aumento da espessura e alteração da textura; positivo para textura se o tendão apresentar sensibilidade à palpação.

\subsubsection{Sinal do Arco}

O teste do Sinal do arco é realizado para verificar se há tendinopatia do tendão do TC. A posição de avaliação foi com o voluntário deitado em decúbito ventral, com os joelhos estendidos e os tornozelos livres. Foi solicitada ao participante para que realizasse a dorsiflexão do tornozelo, avaliando se um arco se formaria, e posteriormente, foi solicitado o movimento de dorsiflexão e flexão plantar, no qual o ventre do tendão era palpado para verificar a presença de inchaço que evidenciasse um edema (MAFFULLI et al., 2003). O resultado era considerado positivo quando havia presença deste sinal.

\subsubsection{Test Hospital Royal London}

Este teste é realizado para verificar se há sensibilidade do TC. A posição de avaliação foi com o voluntário deitado em decúbito ventral, com os joelhos estendidos e os tornozelos livres. Foi solicitado ao voluntário o movimento de dorsiflexão e flexão plantar máxima do tornozelo. Durante a realização, foi questionado ao voluntário se houve dor. O teste é positivo na presença de dor (MAFFULLI et al., 2003).

\subsection{Análise dos dados}

Os resultados foram tabulados no software Microsoft Office Excel $2010 \AA$, onde 
dados foram expressos em média e desvio padrão. A análise dos resultados foi realizada com o programa GraphPad Prism $5.0 \AA$, onde foi feito o tratamento estatístico utilizado o teste $t$ student para a comparação entre os dois grupos analisados (GT vs GC), considerado estatisticamente significativo às comparações com $p \leq 0,05$.

\section{RESULTADOS}

28 voluntários participaram do estudo. Divididos entre 2 grupos: GC (26,8 anos $\pm 8,93)$ e GT $(57,23$ anos $\pm 9,42)$. A tabela 1 demonstra as características dos grupos:

Tabela 1 - Características dos grupos do estudo.

\begin{tabular}{cccc}
\hline Grupo & \multicolumn{1}{c}{ Resumo } & Participantes & Gênero \\
\hline \multirow{2}{*}{ Controle } & $\begin{array}{c}\text { Ausência de doenças } \\
\text { musculoesqueléticas }\end{array}$ & $\mathrm{n}=15$ & Mulheres: 10 \\
& & $\mathrm{n}=13$ & Homens: 5 \\
\multirow{2}{*}{ Tendinopatia } & $\begin{array}{c}\text { Presença de tendinopatia do } \\
\text { TC há mais de 3 meses }\end{array}$ & $\begin{array}{c}\text {-Unilateral no MID: } 4 \\
\text {-Unilateral no MIE: } 2\end{array}$ & Mulheres:4 \\
& & -Bilateral: 7 &
\end{tabular}

$\overline{T C}=$ Tendão do calcâneo; MID= Membro inferior direito; MIE= Membro inferior esquerdo.

Fonte: Os autores.

Para avaliar a morfologia e estrutura do tendão, foi utilizado o recurso do ultrassom de diagnóstico. Na tabela 2 estão demonstrados os resultados quanto à presença ou ausência destes achados.

Tabela 2 - Exame de ultrassonografia.

\begin{tabular}{ccccc}
\hline & \multicolumn{2}{c}{ Grupo Controle } & \multicolumn{2}{c}{ Grupo Tendinopatia } \\
& Presença & Ausência & Presença & Ausência \\
\hline Edema & 0 & $100 \%$ & $59,33 \%$ & $40,67 \%$ \\
Irregularidade & 0 & $100 \%$ & $50,00 \%$ & $50,00 \%$ \\
Circulação intratendínea & 0 & $100 \%$ & $15,5 \%$ & $84,5 \%$ \\
Calcificação & 0 & $100 \%$ & 2,33 & 97,67 \\
\hline
\end{tabular}

Fonte: Os autores.

A tabela 3 demonstra a média geral das medidas da espessura do TC de ambos os membros dos indivíduos do GC e GT. O grupo de tendinopatia foi separado em subgrupos: unilateral no MID (com o membro inferior direito afetado), unilateral no MIE (com o membro inferior esquerdo afetado) e bilateral (ambos os membros com tendinopatia do TC).

Tabela 3 - Medida da espessura do tendão do calcâneo.

(continuação)

\begin{tabular}{cccc}
\hline & & $\begin{array}{c}\text { Espessura }(\mathbf{c m}) \\
\text { Corte longitudinal }\end{array}$ & $\begin{array}{c}\text { Espessura }(\mathbf{c m}) \\
\text { Corte transversal }\end{array}$ \\
\hline Grupo Controle & MID & $0,407 \pm 0,059$ & $0,454 \pm 0,070$ \\
$(\mathbf{n = 1 5})$ & MIE & $0,389 \pm 0,069$ & $0,463 \pm 0,068$
\end{tabular}


Tabela 3 - Medida da espessura do tendão do calcâneo.

(continuação)

\begin{tabular}{cccc} 
Grupo tendinopatia & MID & $0,538 \pm 0,150$ & $0,510 \pm 0,115$ \\
Unilateral no MID $(\mathbf{n}=4)$ & MIE & $0,505 \pm 0,132$ & $0,595 \pm 0,076$ \\
Grupo tendinopatia & MID & $0,460 \pm 0,020$ & $1,000 \pm 0,490$ \\
Unilateral no MIE $(\mathbf{n}=2)$ & MIE & $0,540 \pm 0,080$ & $0,905 \pm 0,285$ \\
Grupo tendinopatia & MID & $0,563 \pm 0,102$ & $0,658 \pm 0,290$ \\
Bilateral $(\mathbf{n}=7)$ & MIE & $0,460 \pm 0,085$ & $0,564 \pm 0,084$ \\
\hline
\end{tabular}

$\mathrm{cm}=$ centímetro; $\mathrm{MID}=$ Membro inferior direito; $\mathrm{MIE}=$ Membro inferior esquerdo. Valores expressos por média \pm desvio padrão.

Fonte: Os autores.

A figura 3 representa o resultado em forma de gráfico da diferença da espessura (imagem obtida no eixo longitudinal) do TC entre os membros. A diferença entre os membros do GT foi superior em $20 \%$, sendo estatisticamente significativa.

Figura 3 - Diferença da espessura do tendão calcâneo entre os membros.

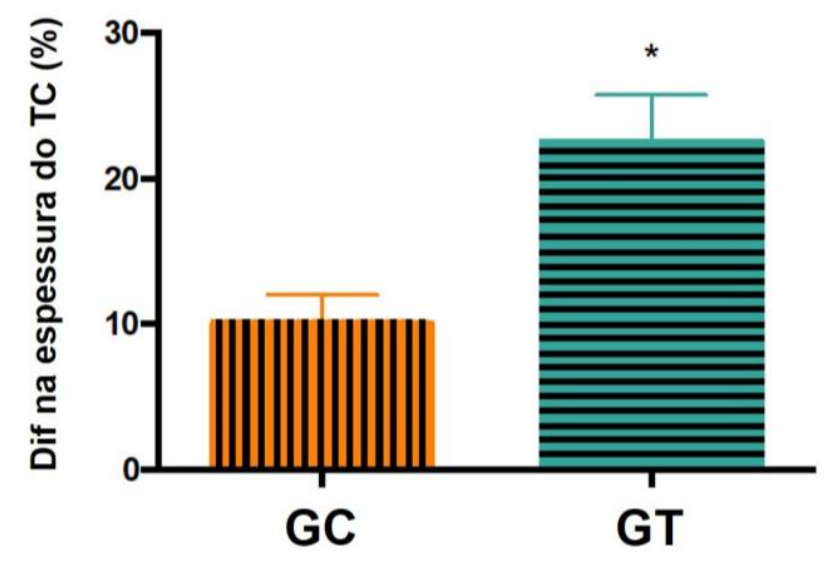

Legenda: Diferença entre espessura do TC. Os valores são representados pelos valores médios e as barras de erro representam o desvio padrão. Dif: diferença; Grupo controle: $G C(n=15)$ e Grupo Tendinopatia: GT $(n=13)$. * $p<0,05$ GT vs GC.

Fonte: Os autores.

Na figura 4 está demonstrado o TC de um participante do grupo controle. A espessura do tendão é simétrica (lado direito $=0,3 \mathrm{~cm}$ e lado esquerdo $=0,33$ ). Já a figura 5 remete ao TC de um voluntário do grupo tendinopatia unilateral no membro inferior direito. Nota-se a assimetria da espessura do tendão. 
Figura 4 - Tendão do Calcâneo de um participante do grupo controle - vista longitudinal.

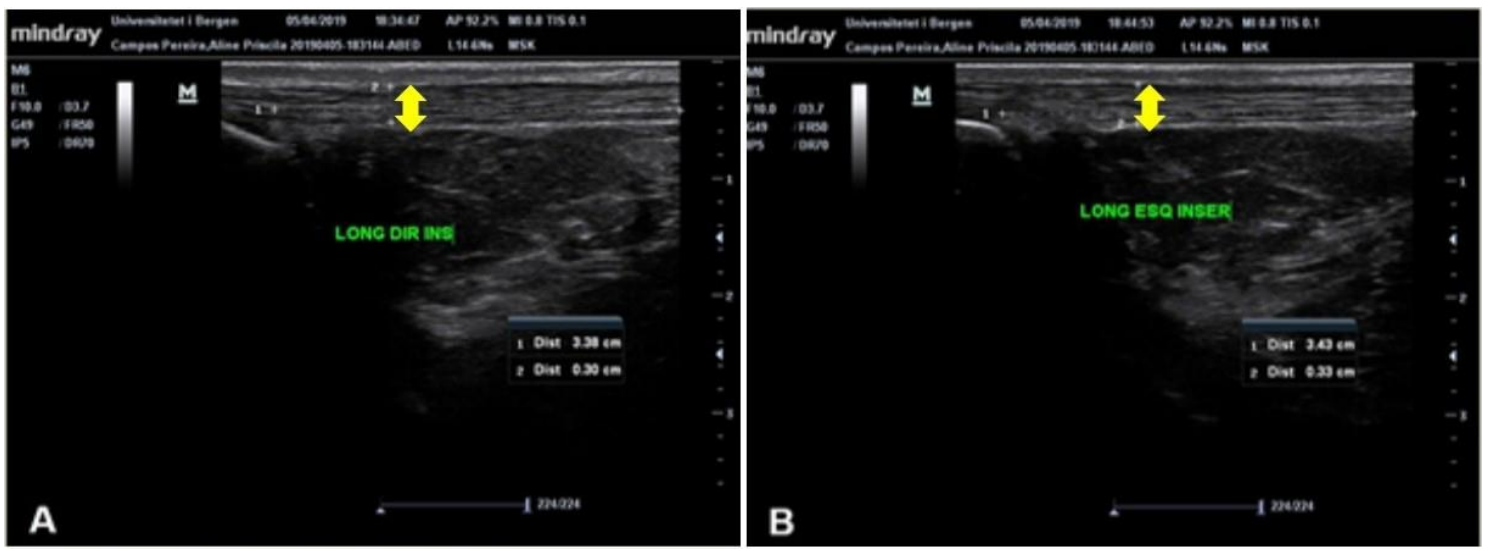

Legenda: $A=$ Membro inferior direito (espessura de $0,3 \mathrm{~cm}$ ); $B=$ Membro inferior esquerdo (espessura de $0,33 \mathrm{~cm}$ ). As setas apontam para as bordas superiores e inferiores, representando o comprimento da espessura do TC.

Fonte: Os autores.

Figura 5 - Tendão do Calcâneo de um participante do grupo tendinopatia (unilateral) - vista longitudinal.

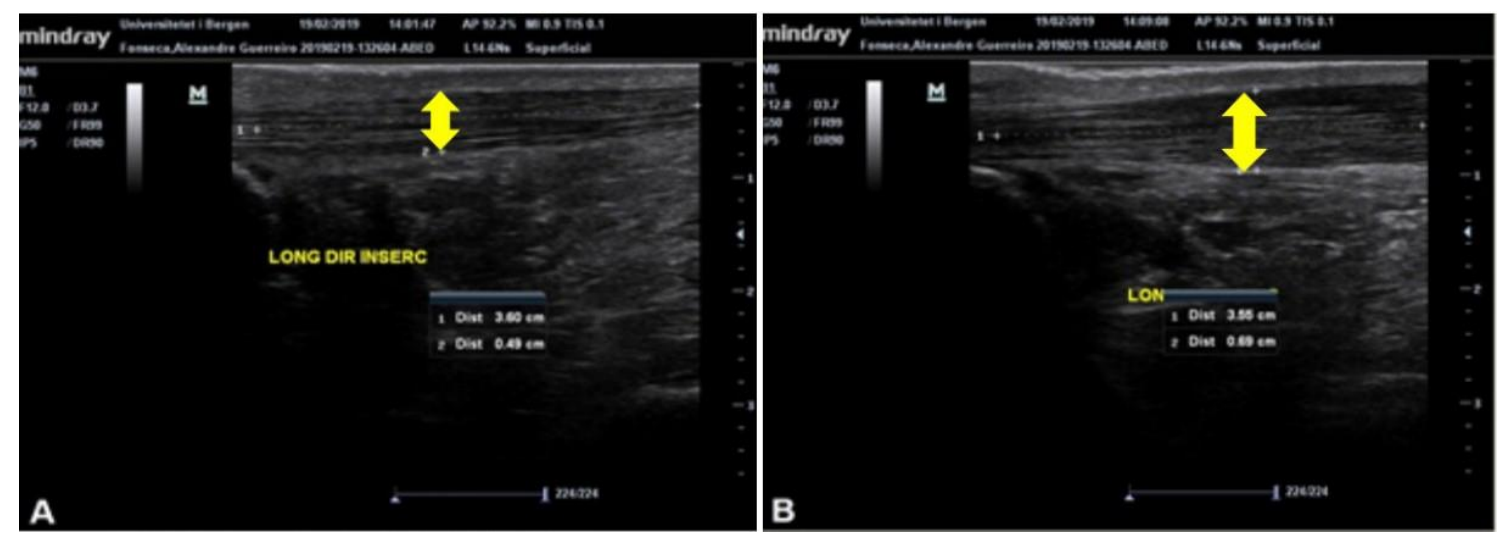

Legenda: $A=$ Membro inferior direito (espessura de $0.49 \mathrm{~cm}$ ); $B=$ Membro inferior esquerdo (espessura de $0.69 \mathrm{~cm}$ ). As setas apontam para as bordas superiores e inferiores, representando o comprimento da espessura do TC.

Fonte: Os autores.

Ainda, o tendão do mesmo participante da figura 5, mas agora no corte transversal (figura 6), observa-se a presença de irrigação intratendínea e peritendínea (pontos arredondados, apontados por setas largas) e edema (regiões hipoecóicas, apontados por setas finas). 
Figura 6 - Presença de edema e irrigação intratendínea no TC com tendinopatia - vista

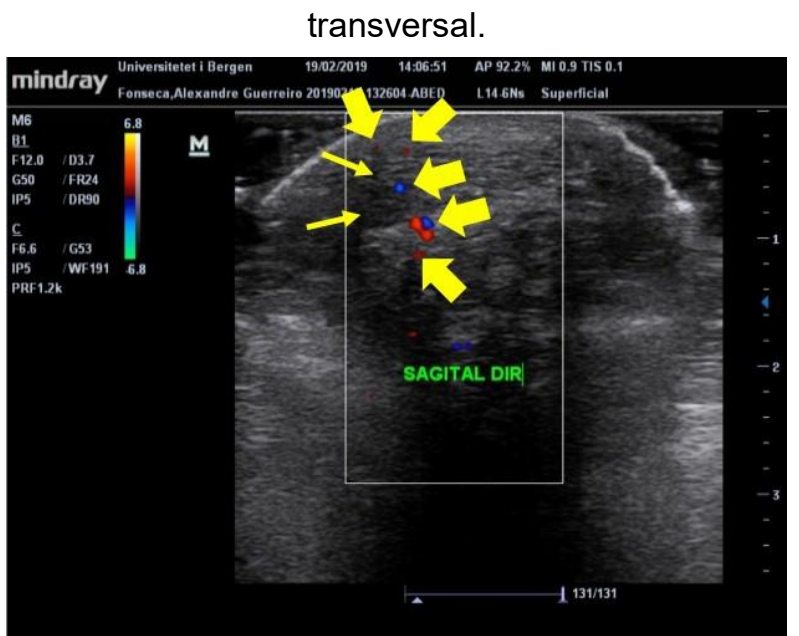

Legenda: Setas largas representam irrigação intratendínea e peritendínea e setas finas apontam para o local de edema.

Fonte: Os autores.

A figura 7 é a imagem obtida do TC de um participante do GT (bilateral). Observase que mesmo na presença de tendinopatia em ambos os membros, é notável a assimetria da espessura entre os tendões (representada pelas setas para cima e para baixo, além dos feixes desorganizados do tendão, irregularidade das bordas (inclusive abaulamento, representado pela seta em curva) e presença de edema (região hipoecóica, representada pelas setas finas).

Figura 7 - Tendinopatia bilateral do TC - vista longitudinal.

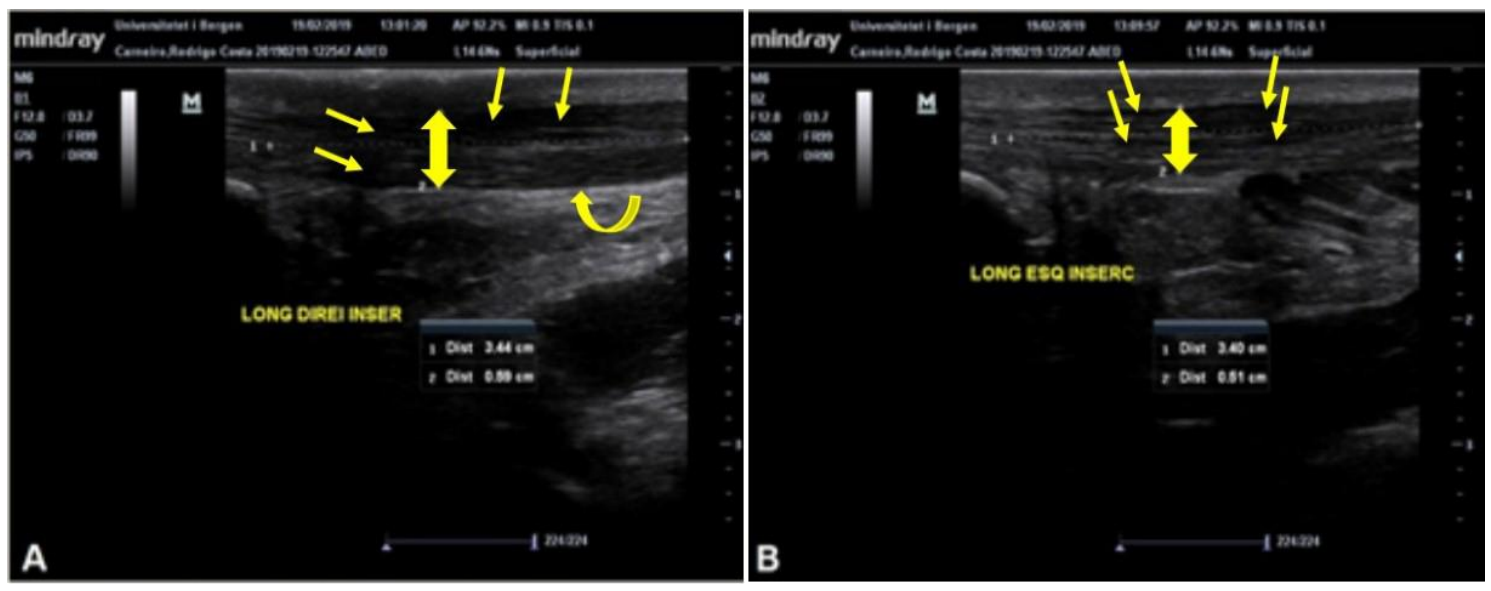

Legenda: $A=$ Membro inferior direito; $B=$ Membro inferior esquerdo. As setas para cima e para baixo apontam para as bordas superiores e inferiores, representando o comprimento da espessura do TC; A seta em curva aponta para o abaulamento do limite inferior do TC; a seta fina aponta para pontos de edema (regiões hipoecóicas).

Fonte: Os autores.

Os próximos resultados são referentes aos testes de palpação e clínicos, também utilizados como avaliação complementar da tendinopatia do TC. Na tabela 4, estão representados os resultados dos testes. Por se tratar de respostas positivo ou negativo, os dados foram apresentados em percentual. 
Tabela 4 - Resultados dos testes à palpação e testes clínicos.

\begin{tabular}{ccccc}
\hline & \multicolumn{2}{c}{ Grupo Controle } & \multicolumn{2}{c}{ Grupo Tendinopatia } \\
& Positivo & Negativo & Positivo & Negativo \\
\hline Crepitação & $6,67 \%$ & $93,33 \%$ & $76,11 \%$ & $23,89 \%$ \\
\hline Tendão Espesso & $6,67 \%$ & $93,33 \%$ & $100 \%$ & 0 \\
\hline Sensibilidade & 0 & $100 \%$ & $50 \%$ & $50 \%$ \\
\hline Sinal do arco & 0 & $100 \%$ & $13,89 \%$ & $86,11 \%$ \\
Test Hospital Royal London & 0 & $100 \%$ & $81,67 \%$ & $18,33 \%$
\end{tabular}

Fonte: Os autores.

\section{DISCUSSÃO}

Foi realizado um estudo transversal com o objetivo de identificar padrões em dois grupos com características distintas. O GC foi composto por indivíduos $(n=15)$ sem diagnóstico de tendinopatia do TC e o GT $(n=13)$ formado por participantes com o diagnóstico de tendinopatia do TC de no mínimo 3 meses.

É importante que estudos com participantes saudáveis, que envolvam também a comparação de simetria corporal, sejam realizados para possibilitar uma medida de comparação do que é normal e patológico, e assim direcionar esforços para prevenções em situações em que há alterações, mas que ainda não geraram sinais clínicos de doenças. Ressaltando, que o achado de assimetria corporal pode indicar uma doença (PAWLOWSKI et al., 2018).

Ao longo da exposição dos resultados, observa-se que as imagens de ultrassom e desfecho dos testes à palpação e os clínicos demonstraram que os participantes do GC tinham o TC saudável, isentos de alterações que indicassem patologia.

Entretanto, os sujeitos do GT apresentaram resultados favoráveis à tendinopatia, como espessamento do tendão, diferença de espessura entre membros, presença de edema e irrigação intratendínea, irregularidades das bordas e da disposição das fibras do TC e sensibilidade na região do TC.

Para traçar o perfil de normalidade da espessura do tendão, mensurou-se o comprimento da espessura do TC dos membros direito e esquerdo de indivíduos sem tendinopatia. A média do comprimento da espessura no corte longitudinal do TC do membro inferior direito e esquerdo foram $0,407 \mathrm{~cm} \pm 0,059 \mathrm{~cm}$ e $0,389 \mathrm{~cm} \pm 0,069 \mathrm{~cm}$, respectivamente.

Alguns estudos da literatura informam que a média do comprimento da espessura do TC saudável é de 0,365cm $\pm 0,055$ (KHARATE; CHANCE-LARSEN, 2012) e 0,550 $\mathrm{cm} \pm 0,04$ (BJORDAL; DEMMINK; LJUNGGREN, 2003). A diferença pode ser devida às diferenças populacionais: uma vez que os trabalhos citados e este estudo em questão terem sido realizados em países diferentes.

Já no GT, a média do comprimento da espessura (também no corte longitudinal) do TC do membro inferior direito variou de $0,460 \mathrm{~cm}$ a $0,563 \mathrm{~cm}$ e do membro inferior 
esquerdo variou de $0,460 \mathrm{~cm}$ a $0,540 \mathrm{~cm}$, considerando as médias separadas em subgrupos de tendinopatia: unilateral no membro inferior direito, tendinopatia unilateral no membro inferior esquerdo e tendinopatia bilateral.

Kharate e Chance-Larsen (2012) trouxeram em seu estudo que a melhor metodologia para avaliar a espessura do TC é no corte longitudinal, pois as imagens no corte transversal superestimam as medidas da espessuras, e ainda há maior probabilidade de erros em medições transversais, pois nesta ocasião, a posição da probe permite mais movimentos, não permanecendo fixa a região avaliada como no caso do corte transversal.

Ainda, sobre o item espessura do TC, também foi realizada a média (em percentual) da diferença entre o comprimento da espessura do TC do membro inferior direito e esquerdo no corte longitudinal. A diferença entre os membros do GT foi superior a $20 \%$, o qual foi estatisticamente significante $(p \leq 0,05)$ em relação ao GC.

Foi demonstrado que em indivíduos com tendinopatia unilateral do TC a diferença do lado comprometido com o lado contralateral sadio foi de aproximadamente $20 \%$ (MUNTEANU, 2015).

Para confirmar a presença da tendinopatia no TC dos indivíduos do GT, também foram realizados testes complementares: teste à palpação e clínicos.

Nas imagens de ultrassom, foi observado que os voluntários do GT apresentaram alterações, demonstrando a presença de edema (59,33\%), irregularidade das bordas $(50,00 \%)$, circulação intratendínea $(15,5 \%)$ e calcificação $(2,33 \%)$. Kharate e ChanceLarsen (2012) relataram em seu estudo que o ultrassom é uma boa técnica de escolha para identificar anormalidades estruturais em tecidos tendíneos.

Os testes à palpação e clínicos (crepitação, espessamento, sensibilidade, Sinal do arco e test Hospital Royal London), específicos para avaliar a presença de tendinopatia do TC também foram positivos para os indivíduos do GT, confirmando mais uma vez a patologia no TC.

Dams e colaboradores (2019) aplicaram testes físicos para aprimorar o diagnóstico e avaliação da tendinopatia no TC, demonstrando que os testes foram importantes na compreensão da perspectiva clínica, relacionadas ao estudo da estrutura e força do tendão, referindo portanto, que mesmo sendo um método de avaliação qualitativo, os testes físicos são válidos para auxílio do diagnóstico de tendinopatia.

Assim, sugerimos que este estudo pode contribuir na prática clínica, quanto ao tratamento preventivo, uma vez que, quando caracterizado um padrão de normalidade, é possível realizar o acompanhamento dos pacientes em relação a condição do TC por meio da ultrassonografia e identificar alterações precocemente. Já que os sintomas 
podem aparecer apenas depois da evolução das lesões, estas que poderiam ser identificadas pelo exame de imagem.

\section{CONCLUSÃO}

Sujeitos saudáveis, sem tendinopatia do tendão do calcâneo, apresentam média do comprimento da espessura no corte longitudinal do TC do membro inferior direito e esquerdo de $0,407 \mathrm{~cm}$ e $0,389 \mathrm{~cm}$, respectivamente. Em indivíduos com tendinopatia do TC, os valores foram maiores, sendo $0,460 \mathrm{~cm}$ a $0,563 \mathrm{~cm}$ e $0,460 \mathrm{~cm}$ a $0,540 \mathrm{~cm}$ para o TC do membro inferior direito e esquerdo, respectivamente.

A diferença da espessura do TC entre membros dos indivíduos do GC foi de $10 \%$, enquanto no GT foi de $22 \%$. Logo, sugere-se que diferenças da espessura do TC superiores a $20 \%$ indicam a presença de tendinopatia, comprovadas pelos achados nas imagens de ultrassom e pelos resultados positivos dos testes à palpação e clínicos.

\section{AGRADECIMENTOS}

À Coordenação de Aperfeiçoamento de Pessoal de Nível Superior - CAPES Código de financiamento 001, pelo apoio financeiro.

\section{REFERÊNCIAS}

BENJAMIN, M. et al. Where tendons and ligaments meet bone: attachment sites ('entheses') in relation to exercise and/or mechanical load. Journal of Anatomy, v. 208, n. 4, p. 471-490, 2006.

BJORDAL, J. M.; DEMMINK, J. H.; LJUNGGREN, A. E. Tendon Thickness and Depth from Skin for Supraspinatus, Common Wrist and Finger Extensors, Patellar and Achilles Tendons. Physiotherapy, v. 68, n. 6, p. 375-383, 2003.

CHINESE SOCIETY OF SPORTS MEDICINE. Chinese Consensus on Insertional Achilles Tendinopathy. The Orthopaedic Journal of Sports Medicine, v. 7, n. 10, p. 16, 2019.

COOK, J. L.; KHAN, K. M.; PURDAM, C. Achilles tendinopathy. Manual Therapy, v. 7, n. 3, p. 121-130, 2002.

DAMS, O. C. et al. The recovery after Achilles tendon rupture: a protocol for a multicenter prospective cohort study. BMC Musculoskeletal Disorders, v. 20, n. 1, p. 69, 2019.

HASLERUD, S. et al. Achilles Tendon Penetration for Continuous $810 \mathrm{~nm}$ and Superpulsed $904 \mathrm{~nm}$ Lasers Before and After Ice Application: An In Situ Study on Healthy Young Adults. Photomedicine Laser Surgery, n. 35, p. 10, p. 567-575, 2017.

JÓZSA, L. G.; KANNUS, P. Human Tendons: Anatomy, Physiology and Pathology. Champaign: Human Kinetics, 1997. 
KHAN, K. M. et al. Histopathology of common tendinopathies: update and implications for clinical management. Sports Medicine, v. 27, n. 6, p.393-408,1999.

KHARATE, P.; CHANCE-LARSEN, K. Ultrasound evaluation of Achilles tendon thickness in asymptomatic's: A reliability study. International Journal of Physiotherapy and Rehabilitation, v. 2, p. 1-11, 2012.

KRAEUTLER, M. J.; PURCELL, J. M.; HUNT, K. J. Chronic Achilles Tendon Ruptures. Foot Ankle International, v. 38, n. 8, p. 921-929, 2017.

MAFFULLI, N. et al. Clinical diagnosis of Achilles tendinopathy with tendinosis. Clinical Journal of Sport Medicine, v. 13, n. 1, p. 11-15, 2003.

MAFFULLI, N.; SHARMA, P.; LUSCOMBE, K. L. Achilles tendinopathy: aetiology and management. Journal of the Royal Society of Medicine, v. 97, n. 10, p. 472-476, 2004.

MARTIN, R. L. et al. Achilles Pain, Stiffness, and Muscle Power Deficits: Midportion Achilles Tendinopathy Revision 2018. Journal of Orthopaedic \& Sports Physical Therapy, v. 48, n. 5, p. A1-A38, 2018.

MASCI, L. et al. How to diagnose plantaris tendon involvement in midportion Achilles tendinopathy - clinical and imaging findings. BMC Musculoskeletal Disorders, v. 17, p. $97,2016$.

MENZ, H. B. et al. Characteristics of primary care consultations for musculoskeletal foot and ankle problems in the UK. Rheumatology, v. 49, n. 7, p. 1391-1398, 2010.

MUNTEANU, S. Achilles Tendon. In: ROME, K.; MCNAIR, P.; NESTER, C.

Management of Chronic Conditions in the Foot and Lower Leg. London: Elsevier, 2015. P. 145-179.

O'BRIEN, M. The anatomy of the Achilles tendon. Foot Ankle Clinical, v. 10, n. 2, p. 225-38, 2005.

PAWLOWSKI, B. et al. Human body symmetry and immune efficacy in healthy adults. American Journal of Physical Anthropology, v. 167, n. 2, p. 207-216, 2018.

RAMACHANDRAM, M. Basic orthopaedic sciences: the Stanmore guide. London: Hodder Arnold, 2007. 304 p.

RIO, E. et al. The Pain of Tendinopathy: Physiological or Pathophysiological? Sports Medicine, v. 44, n. 1, p. 9-23, 2014.

RYAN, M. et al. Kinematic analysis of runners with achilles mid-portion tendinopathy. Foot \& Ankle International, v. 30, n. 12, p. 1190-5, 2009.

SOUZA, N. S. S.; SANTANA, V. S. Cumulative annual incidence of disabling workrelated musculoskeletal disorders in an urban area of Brazil. Cadernos de Saúde Pública, v. 27, n. 11, p. 2124-2134, 2011.

VIEIRA, F. et al. Tendinopatia do tendão calcâneo. Publicatio UEPG: Biological and Health Sciences, v. 16, n. 1, p. 35-42, 2010. 